\title{
Investigación educativa y gestión escolar: ¿Un binomio ejemplar?
}

SILVIA SCHMELKES ${ }^{2}$

Departamento de Investigaciones Educativas/CINVESTAV

\section{INTRODUCCIÓN}

(3) iempre ha interesado tanto a investigadores como a tomadores de decisiones, aunque probablemente bastante más a los primeros, la manera de vincular mejor y más eficientemente los resultados de la investigación con la toma de decisiones en materia educativa. En el campo de la gestión escolar esta relación se ha dado casi por definición, como veremos más adelante. Propongo compartir con ustedes la manera como, en este campo, la investigación educativa ha generado hipótesis y preguntas de investigación que, surgidas desde los maestros y los tomadores de decisiones, han orientado un conjunto de investigaciones educativas muy vinculadas a la práctica escolar. Analizaremos los avances y las limitaciones de este campo de investigación educativa, nos referiremos a los retos que conviene enfrentar en el próximo futuro, y comentaremos sobre la relación entre investigadores, docentes y tomadores de decisiones en este campo.

1. Conferencia presentada en el Congreso Regional de Investigación Educativa, celebrado en la Ciudad de Puebla, Pue., del 17 de septiembre de 1997.

2. La Mtra. Silvia Schmelkes es investigadora del Dpto. de Investigaciones Educativas del cinvESTAV y es tutora del Doctorado Interinstitucional en Educación, programa coordinado por la LAA. 
En la década de los sesentas, cuando las enormes computadoras de la primera generación se comenzaron a utilizar en las ciencias sociales, se hicieron varios estudios sobre los factores que explicaban el nivel escolar alcanzado por alumnos que venían de diversos tipos de familia (el más conocido de estos estudios, por la magnitud de su muestra, fue el llamado Informe Coleman ${ }^{3}$, realizado en Estados Unidos). Estos estudios parecían llegar a la conclusión de que el factor más importante para explicar la trayectoria de los niños en la escuela y el nivel educativo alcanzado era el socioeconómico. Parecía, entonces, que era poco lo que la escuela podía hacer para modificar un futuro que casi aparecía como fatal.

Curiosamente, fueron los maestros los que se propusieron probar precisamente lo contrario: que la escuela era capaz de asegurar el éxito escolar de todos los alumnos, independientemente de la clase social de la que procedían. Fue así como se inicia el llamado "movimiento de las escuelas efectivas". Este movimiento promueve, a partir de los años setentas y hasta la fecha, el estudio de escuelas que efectivamente han logrado el éxito escolar de alumnos procedentes de familias de nivel socioeconómico desfavorecido.

Coleman y otros autores no se equivocaron del todo. En realidad, el peso del origen socioeconómico y, como más adelante se ha venido demostrando, del cultural ${ }^{\dagger}$, es efectivamente muy fuerte. Esto, sin

3. Coleman, J. S., E. Camphell, C. Hobson, J. McPartland, A. Mood, F. Weinfield, y. R. York Equality of Educational Opportunity: Washington: US Government Printing Office, 1966.

4. Por origen cultural me refiero aquí fundamentalmente tanto a la escolaridad de los padres como a la importancia que en la familia se le brinda a la asistencia y al progreso escolar de los hijos. Especialmente importante en la documentación de la variable cultural es, para América Latina, el estudio de Germán Rama, Qué Aprenden y Quiénes Aprenden en las Escuelas de Uruguay. Montevideo: CEPAL. 1991. Este estudio demuestra que la escolaridad media de la madre (primaria completa o secundaria). incluso en situaciones socioeconómicas desventajosas, actúa como vacuna contra el fracaso escolar. 
embargo, no significa que no haya nada que hacer desde la escuela. Por el contrario, lo que significa es que las escuelas tienen que hacer un esfuerzo sobresaliente para obtener buenos resultados. El movimiento de escuelas efectivas muestra que estos esfuerzos sí se dan, y que cuando se dan, se consiguen resultados exitosos con los alumnos.

Los estúdios realizados desde esta perspectiva se han multiplicado notablemente en muchos países del mundo. Las metodologías para estudiar las escuelas efectivas se ha venido complejizando y sofisticando, de manera que se han vuelto mucho más rigurosas. El conocimiento acumulado a la fecha es enorme, y abundan las evidencias de escuelas que, con todos los factores en su contra, logran trayectorias escolares y resultados de aprendizaje, entre sus alumnos, incluso superiores a las que atienden a alumnos de familias de clase media y alta.

\section{Algunos RESULTAdos DE LOS ESTUdios DE ESCUELAS EFECTIVAS}

El carácter casuístico de muchos de los estudios sobre escuelas efectivas ha conducido a la realización de múltiples "estados del arte" sobre el tema -intentos realizados por los investigadores para encontrar patrones en los factores que aparecían reportados en los diversos estudios empíricos como relacionados con la efectividad escolar. En algún momento, se llegó inclusive a decir que existían más meta-estudios sobre el tema que estudios empíricos originales. Si bien podemos afirmar que efectivamente hay en este campo un número exagerado de "estudios sobre estudios", quizás gracias a ello nos resulta tan fácil ahora trazar la peculiar historia de este campo de la investigación educativa.

De la primera época de estos estudios, llevada a cabo fundamentalmente en los Estados Unidos pero con importantes ramificaciones en varios países europeos, surge un "modelo de cinco factores", factores que se encontraban, todos ellos, presentes en escuelas que 
mostraban una efectividad extraordinaria en relación a otras escuelas de niveles socioeconómicos y culturales similares. Estos cinco factores eran los siguientes:

1) Fuerte liderazgo académico.

2) Altas expectativas sobre el logro académico de los alumnos.

3) Énfasis de la escuela en la adquisición de habilidades básicas.

4) Un clima escolar seguro, ordenado y propicio al aprendizaje.

5) Una evaluación frecuente, con retroalimentación, del avance académico de los alumnos.

Estos cinco factores, como señalamos, surgen de su presencia en la mayor parte de los estudios llevados a cabo en escuelas exitosas, o en estudios que comparaban escuelas exitosas con escuelas "fracasadas" y/o en escuelas "típicas", siempre manteniendo constante el contexto.

Con el tiempo, estos factores se han ido complejizando. Así, por ejemplo, un meta-estudio reciente ${ }^{6}$ realizado sobre estudios conducidos durante la última década en Inglaterra señala como los factores presentes en todos los estudios que los toman en cuenta los siguientes:

1) Liderazgo profesional. Según estos estudios, el liderazgo debe ser firme y claro. El enfoque del liderazgo debe ser participativo. El liderazgo debe ser académico, es decir, el líder tiene que ser un educador, tiene que saber lo que ocurre en las aulas y lo que hacen los maestros, tiene que estar al tanto de los apoyos que requieren los

5. Ver B. M. P. Creemers, "History, Value and Purpose of School Effectiveness Studies", en D. Reynolds, et al. (eds.). Advances in School Effectiveness Research and Practice. Oxford: Pergamon, 1994.

6. Ver Sammons, P., J. Hillman y P. Mortimore. Key Characteristics of Effective Schools: A Review of School Effectiveness Research. Londres: International School Effectiveness and Improvement Center, Institute of Education, University of London, 1995. 
docentes. En síntesis, se trata de un liderazgo centrado en los procesos de enseñanza y aprendizaje.

Como vemos, este factor, presente desde los primeros estudios permanece, y es de los que con mayor consistencia se encuentra en los estudios que lo toman en cuenta. Donde hay una buena escuela, hay un buen director.

2) Visión y metas compartidas. El equipo de trabajo, y también los alumnos, comparten una visión de lo que quieren lograr, para lo cual sistematizan un conjunto de prácticas que llevan a cabo de manera consistente. Una parte importante de las actividades de la escuela se lleva a cabo de manera colaborativa y con espíritu de colegialidad. Dichas actividades pueden involucrar no sólo al personal de la escuela, sino también a docentes y a padres de familia. El trabajo en equipo de los docentes aparece como una condición siempre presente en las escuelas efectivas.

Cabe señalar que en América Latina la investigación sobre escuelas efectivas comenzó hace muy poco, aunque está cobrando enormes bríos. No obstante, en dos estudios de principios de la década, uno en México y otro en Uruguay, aparece la importancia de que la escuela logre transmitir esta visión y estas metas a la comunidad: que la escuela sea capaz de enviar mensajes claros acerca de lo esperado y de lo inadmisible $e^{i}$.

3) Un ambiente de aprendizaje. Sigue apareciendo este factor como importante. Las escuelas que tienen buenos resultados de aprendizaje entre sus alumnos de clases populares generalmente cuentan con un ambiente ordenado. Se añade a esta característica el hecho de que dicho ambiente es agradable y atractivo. Estas escuelas priorizan los momentos para aprender. No se dejan interrumpir. Por otra parte, el acto de aprender se hace placentero, divertido, fas-

7. Ver Christopher James Martin, "Para Poderse defender en la Vida: Cuestiones sobre la Cultura Educativa de Familias Obreras en el Occidente de México", en Revista Latinoamericana de estudios Educativos, Vol. XXI, No. 43 (invierno), 1991, y Roma, op. cit. 
cinante. El aprendizaje real se convierte en el antídoto más efectivo a la indisciplina. Lo contrario parece también ser cierto: en una escuela en la que es difícil prever lo que va a ocurrir, en la que el ruido alcanza niveles incontrolables, donde la indisciplina irrumpe en el quehacer académico, los resultados de aprendizaje no pueden ser buenos.

4) Enseñanza con propósitos claros. Los maestros saben a dónde van con su enseñanza. Planean el día, la semana, el mes. Se organizan de manera eficiente para cubrir las actividades previstas. Las lecciones están estructuradas y el proceso y los objetivos son claros para los alumnos. El curriculum oficial se adapta a las características de los alumnos y a los eventos importantes -rara vez se sigue al pie de la letra-. Estudios en América Latina han descubierto, además, la importancia de que el maestro organice su clase de manera que pueda brindar atención individual a los alumnos que lo necesitan ${ }^{8}$. Asimismo, el trabajo en equipo, donde los alumnos trabajan por sus cuenta en pequeños grupos -mejor si son heterogéneos-, además de permitir lo anterior, produce mejores resultados de aprendizaje.

5) Altas expectativas. Otro de los factores que permanece, y con un gran peso. Quizá de todas las variables, ésta sea la que con mayor consistencia aparece como importante en prácticamente todos los estudios que la incluyen. Tiene que ver con el hecho de que el director crea en su personal, de que los maestros crean en sus alumnos, y con que estas altas expectativas que se tienen respecto a los logros potenciales se comuniquen de manera efectiva.

8. Remito al auditorio al estudio pionero coordinado por Carlos Muñoz Izquierdo en el año de 1979 y publicado bajo el título "El Síndrome del Atraso Escolar", en Revista Latinoamericana de Estudios Educativos, Vol. IX, No. 3 (otoño). Este estudio descubre que el fracaso escolar es consecuencia del rezago escolar progresivo, provocado por el hecho de que el maestro no brinda atención individual a los alumnos. La reprobación. que podría prevenirse. es una decisión que se toma al final del ciclo escolar cuando ya no es posible resolver el problema. 
Ahora bien, la presencia de altas expectativas debe traducirse en un programa que permita presentar desafíos continuos a alumnos y maestros. Sólo de esta forma, además de mantener el interés de los alumnos en su proceso de aprendizaje, podrán mostrarse de manera periódica los avances y retroalimentar las expectativas, que deberán terminar siendo altas para cada uno de los integrantes de la comunidad escolar respecto de sí mismo.

Vinculado a las altas expectativas, añadiríamos la necesaria valorización de la cultura de la comunidad por parte del personal de la escuela. Sobre todo en las sociedades en las que existe discriminación por motivos raciales o de extracción cultural, está demostrado que la presencia de lo contrario -discriminación y desvaloración de la cultura de los alumnos- explica su fracaso escolar y su eventual deserción".

6) Refuerzo positivo. Muy ligada a la variable anterior se encuentra la presencia, en las escuelas efectivas, de prácticas consistentes de retroalimentación positiva a los alumnos de parte de los maestros y a los maestros de parte del director. En el caso de los alumnos, se habla de las ventajas de una disciplina clara y justa, basada mucho más en la pertinencia y la participación que en las reglas y controles. Las cosas se hacen bien porque son para nosotros, en nuestra institución. La retroalimentación, por su parte, debe darse cuando hay motivos para alentar, no cuando hay razones para desalentar. Y nunca deben darse refuerzos positivos cuando no hay acciones por parte del sujeto que los ameriten.

7) Monitoreo de los avances. Este es otro de los cinco factores originales. La capacidad de una escuela de dar cuenta con relativa objetividad del avance de los alumnos, de los maestros y de la escuela como un todo se encuentra claramente vinculada a su efectividad medida en términos de logro de aprendizaje entre sus alum-

9. Ver la literatura sobre violencia simbólica, especialmente Bernstein, Willis. Bourdieu. 
nos. Dicho monitoreo supone planeación. También supone la existencia de reuniones periódicas del personal de la escuela. Ahora bien, es importante señalar que no basta con monitorear si los resultados de dicho monitoreo no se incorporan al proceso de toma de decisiones. Entre las decisiones más importantes consecuencia de los monitoreos se encuentra el desarrollo. (superación, actualización) del personal. Agregaríamos bajo este rubro la evaluación de resultados que, al terminar un ciclo, permite rendir cuentas a la comunidad con la que, idealmente, se han compartido metas anuales.

8) Participación de los alumnos. Este conglomerado de variables incluye aspectos tales como la promoción de la autoestima entre los alumnos, la que a su vez depende mucho de una cálida y respetuosa relación de los maestros con los alumnos. Incluye también el otorgamiento de muchas oportunidades de participación a los alumnos en posiciones y actividades que supongan responsabilidad y, entre ellas, responsabilidad sobre otros. Algunas de estas oportunidades deben referirse al trabajo escolar mismo.

9) Relación con la familia. Esta variable, que no aparecía en los cinco factores originales, emerge en la segunda generación de estudios sobre escuelas efectivas como absolutamente central. Junto con la variable "altas expectativas" y "liderazgo profesional", ésta se relaciona de forma notablemente consistente con altos niveles de aprendizaje en los diversos estudios que la han considerado. Dicha relación se da de múltiples maneras: desde que los padres entiendan la importancia de enviar a sus hijos a la escuela regularmente, hasta que asuman algunas responsabilidades de carácter educativo al interior de la misma. El tipo de relación que pueda establecerse dependerá del nivel cultural del medio y de la edad de los alumnos. Lo importante, sin embargo, es que cada escuela y familia se vuelvan aliados efectivos en torno al aprendizaje y el logro escolar de los niños. Investigaciones latinoamericanas permiten señalar que la presencia de la escuela en la comunidad, y no sólo en la familia, también es importante. La rěalización de actividades 
que invadan directa o indirectamente la comunidad (por ejemplo, tareas escolares que implican preguntar o servir a la comunidad) se encuentra en este caso claramente asociada con mayores logros académicos ${ }^{10}$.

10) Una organización de aprendizaje. La escuela es una organización en la que todos aprenden, incluyendo a los docentes y a los directivos. Es cuando este aprendizaje se da en la escuela misma, y en función de lo que sus miembros necesitan para lograr mejores niveles de aprendizaje de sus alumnos -y en este sentido se habla de una organización de aprendizaje- que la superación del personal se traduce en mayor aprendizaje de los alumnos. Se trata, por otra parte, de procesos de superación que son continuos y ascendentes, y que se vinculan con los procesos de planeación y de revisión de lo planeado.

En síntesis, se conocen ya muchas de las cosas que ocurren en las escuelas que, trabajando en situaciones desventajosas, logran resultados educativos extraordinarios. Así, por ejemplo, se sabe que en estas escuelas existe un fuerte liderazgo académico de parte del director. Este liderazgo no es autoritario, sino participativo. Privilegia el involucramiento y la continua formación del equipo docente. En estas escuelas, por otra parte, se trabaja en equipo. Existe una cultura de planeación tanto de la escuela como un todo, como del trabajo en el aula. Las escuelas efectivas tienen un clima propicio al aprendizaje. Son agradables. Los alumnos asisten con gusto y permanecen contentos. Hay muchas oportunidades para que éstos participen, tanto en su propio aprendizaje como en actividades de responsabilidad sobre los demás. Estas escuelas mantienen una estrecha relación con los padres de familia y con la comunidad. Lo que se persigue es establecer una alianza entre padres y maestros,

10. En México encontramos esta relación virtuosa en zonas rurales desde 1979 (Ver S. Schmelkes, et al. "Estudio Exploratorio sobre la Participación de la Comunidad en la Escuela Rural Básica Formal", en Revista Latinoamericana de estudios Educativos, Vol. Ix, No. 4. 
entre comunidad y escuela, para lograr que los niños aprendan más y mejor. Así, las escuelas tienen normas claras y son capaces de comunicar a padres y alumnos el mensaje de lo que la escuela espera y de lo que no permite. En estas escuelas hay evaluaciones frecuentes, tanto de los alumnos como de lo que la escuela plané́. Estas evaluaciones sirven para retroalimentar a los alumnos y a la comunidad educativa, de forma tal que constituye un insumo para mejorar.

Este movimiento que iniciaron los maestros, molestos porque su quehacer parecía fortuito, ha logrado demostrar que la escuela sí puede hacer la diferencia. Se sabe ahora que ya no hay excusa para el fracaso escolar, porque éste casi siempre se puede evitar.

Lo anterior es una muestra de los resultados de la investigación sobre escuelas efectivas. A continuación analizaremos algunas de sus limitaciones.

\section{LAS CRÍTICAS A LA INVESTIGACIÓN SOBRE ESCUELAS EFECTIVAS}

La investigación sobre escuelas efectivas ha sufrido, en sus diversas etapas, serias críticas por parte de la comunidad de investigadores de la educación, incluso de parte de aquellos interesados en su desarrollo. Quizás entre las más importantes se encuentren tres a las que me referiré a continuación.

1) Además de las críticas a los estudios específicos, sobre todo los de la primera generación, relativas a los procedimientos de muestreo y a la metodología de medición, una de las críticas más severas que se hacen a este campo de la investigación es el hecho de que sus resultados son sólo correlatos de la efectividad escolar. En este sentido, si bien parecen asumir causalidad, estrictamente en ningún caso se puede afirmar que existe una relación causal entre las variables identificadas y el aprendizaje excepcional de los alumnos. Incluso, se dice, es posible suponer que la relación causal es invertida. Así, por ejemplo, quizás las altas expectativas 
sean el resultado, y no la causa del aprendizaje excepcional de los alumnos.

Justamente por el hecho de que las relaciones establecidas no son de causalidad, no deben ser mecánicamente utilizadas por los tomadores de decisiones que lo que persiguen es mejorar la efectividad de las escuelas. Desafortunadamente, este es el primer uso que se le ha dado a los resultados de escuelas efectivas. Pero sobre eso volveremos enseguida.

2) La investigación sobre escuelas efectivas ha preferido el estudio de las variables a nivel escuela, cuando bien se sabe que las variables que más potencialidad tienen de afectar el aprendizaje de los alumnos tienen que ver con lo que ocurre en el aula. La limitación, al parecer, se ha debido fundamentalmente a la ausencia de instrumental suficientemente fino para categorizar y medir lo que ocurre en el aula.

Por fortuna, ha habido un interesante desarrollo, más reciente, acerca de lo que se ha dado en llamar "aulas efectivas"". Más interesante aún, hay un creciente acercamiento de ambas líneas de investigación: la de escuela y la de aula. No obstante, puede afirmarse que hasta la fecha contamos con más información acerca de la gestión escolar que acerca de la administración y práctica de la enseñanza para intentar explicar el éxito escolar.

3) La investigación sobre escuelas efectivas resulta simplista. La realidad del cambio escolar es mucho más compleja: el cambio escolar es multidimensional, lento, no lineal ${ }^{12}$. Por otra parte, los factores explicativos del éxito o del fracaso escolar se encuentran ubicados en múltiples niveles y con una estrecha relación entre sí: a nivel de los alumnos, a nivel del aula, a nivel de la escuela, a nivel

11. Ver, por ejemplo, Slavin, Robert E. "Salas de Clase Efectivas, Escuelas Efectivas: Plataforma de Investigación para una Reforma Educativa en América Latina". Santiago: Documentos PREAl, 1996.

12. Esta visión es sostenida por la llamada "Escuela Canadiense de Efectividad Escolar", entre cuyos exponentes se encuentran Fullan y Hargreaves. 
del contexto circundante, y -algo curiosamente olvidado por estas investigaciones- a nivel del sistema educativo, desde la zona hasta la cúpula. Es necesario complejizar los modelos para entender mejor el éxito y el fracaso escolar. Y esta necesidad nos conduce a la siguiente crítica.

4) La investigación sobre efectividad escolar es eminentemente empírica. Se ha construido a partir de la acumulación de información procedente de los casos de escuelas exitosas, y de éstas comparadas con sus pares no tan exitosas o de plano fracasadas. Carece de marcos teóricos de análisis y como tal de toda posibilidad de explicación. Los meta-estudios buscan fundamentalmente encontrar las coincidencias fundamentales entre los diversos estudios realizados, pero ellos también se encuentran desprovistos de marcos teóricos capaces de ofrecer explicaciones que puedan conducir, de manera efectiva, al cambio escolar.

Estas críticas han tenido efectos sobre el movimiento de escuelas efectivas. De las primeras críticas relativas a la falta de rigor muestral y metodológico surgieron investigaciones mucho más rigurosas. De las críticas a la falta de importancia atribuida a lo que ocurría en el aula surge todo un movimiento crecientemente articulado con el primero sobre aulas efectivas. De las críticas al relativo simplismo de las preguntas de investigación surgen modelos multiniveles que complejizan la visión sobre el éxito escolar. Estos modelos, además de incluir otros niveles de análisis, establecen relaciones entre factores de los diversos niveles de análisis y plantean relaciones no sólo causales, sino también recíprocas.

Las críticas también han propiciado una seria búsqueda de sustento teórico al estudio del cambio escolar. Aquí hay que reconocer que el avance ha sido bastante menor que en relación con las respuestas de la comunidad de investigadores a las otras críticas. Los modelos multinivel de alguna manera suponen un recurso mayor a la teoría, junto con una recuperación de planteamientos teóricos de las ciencias de la educación (de teorías de la enseñanza, por 
ejemplo, para entender lo que ocurre en el aula). Otras aproximaciones han planteado la necesidad de recurrir a la teoría de la contingencia (lo que ocurre en la escuela es contingente con el espacio y el tiempo que rodean la escuela específica -tiene éxito lo que responde a ese contexto), o incluso a la teoría del caos, para explicar el cambio escolar exitoso. Es necesario reconocer que los avances son aún endebles, y que seguramente en este aspecto encontramos el principal reto al desarrollo futuro de este campo de la investigación educativa.

\section{UNA ADVERTENCIA SOBRE EL USO DE LA INVESTIGACIÓN SOBRE EFECTIVIDAD ESCOLAR}

Hacíamos referencia un poco más arriba a la utilización mecánica de los resultados de la investigación sobre efectividad escolar por parte de algunos tomadores de decisiones, que parecen considerar que, si ya se sabe lo que está presente en las escuelas exitosas, sólo basta con incorporar estas variables en las escuelas no exitosas.

Sin duda, la investigación sobre escuelas efectivas nos ilumina a todos respecto de lo que debe haber en una escuela para que pueda obtener mejores resultados con sus alumnos. Pero considerar que los resultados pueden convertirse mecánicamente en soluciones es evidentemente un error. Quizás una de las razones importantes por la que podemos sostener que esto es un error se encuentra justamente en aquellos factores que no coinciden entre los estudios, que son muchos más que los que sí coinciden. Ello es lo que conduce a sostener que no hay escuelas típicas, que cada escuela es única y que las verdaderas escuelas exitosas son aquellas que han logrado tomar decisiones, adoptar las medidas y transformar las prácticas que esa escuela necesita para tener éxito con esos alumnos y en ese contexto específico. De esta forma, la solución consiste en que la escuela adquiera la capacidad para adaptarse de manera dinámica al contexto en una búsqueda continua del logro de mayor calidad. 
Otra importante razón por la cual puede resultar peligrosa la transferencia acrítica de los resultados de la investigación a la política educativa es el hecho de que pueden no tomarse en cuenta las dos dimensiones de la calidad: el logro de buenos resultados de aprendizaje, y el logro de la equidad. Muchos de estos estudios se han basado en una noción de efectividad que se limita a la primera dimensión de calidad. Eso significa que la escuela selecciona a fin de lograr, entre los alumnos que sí pueden, excelentes resultados de aprendizaje. Pero lo que se pretende, si se persigue la calidad en sus dos dimensiones, es lograr aprendizaje efectivo con equidad. Desde esta segunda perspectiva, los resultados de la investigación tendrían que analizarse con mucho mayor cuidado.

Se ha presentado el caso de algunos gobiernos que han "saboteado" -según dicen sus críticos- la investigación sobre las escuelas efectivas. Funcionan bajo la siguiente lógica: si hay escuelas que han demostrado que pueden lograr buenos resultados de aprendizaje, entonces las otras escuelas tienen la culpa de no lograrlo. Y se les castiga. Ello ha conducido a un proceso perverso mediante el cual directores y maestros efectivamente comprometidos resultan completamente desmoralizados y abandonan sus esfuerzos.

Efectivamente, si se define a la escuela "fracasada" sólo como aquella que rinde por debajo de los estándares, y se "castiga" de alguna manera a estas escuelas que fracasan -como ocurrió durante algún tiempo en Inglaterra- se pueden cometer graves errores. Porque no es lo mismo una escuela que "fracasa" y va descendiendo que otra que "fracasa", pero va en ascenso. Tratarlas de la misma manera es un acto de injusticia.

Por último, los sistemas educativos han tendido a utilizar los resultados de la investigación sobre escuelas efectivas para propiciar el cambio en la escuela sin cambiar el sistema. El énfasis que este movimiento le ha dado a las variables a nivel de escuela ha conducido a que se descuiden las variables del sistema que de hecho limitan, o incluso impiden, que el cambio escolar se dé. Se requiere 
un sistema que cambie sus prácticas de forma tal que sea capaz de operar en función de la escuela y de sus necesidades, en un sentido de apoyo y no de control, de aliento y no de culpa.

\section{Comentario FINAL}

Subtitulé esta conferencia con una pregunta relativa a si la relación entre la investigación y la toma de decisiones era ejemplar. Yo solicito al auditorio que aborde esta pregunta en sus comentarios durante el debate. Simplemente señalo, para terminar, que quizás estemos ante una paradoja: en este campo de la investigación, que nació tan vinculado a la práctica escolar y a la toma de decisiones, ¿no será necesario propiciar una mayor distancia entre los resultados de la investigación y quienes deciden?

Independientemente de lo anterior, la investigación sobre las escuelas efectivas ha demostrado con suficiente vigor que el fracaso escolar se puede evitar, que en ello la escuela singular juega un papel indiscutible, y que existen razones para sostener la necesidad de una mayor confianza por parte de los sistemas educativos en los equipos escolares para que éstos tomen las decisiones que procedan para enfrentar con calidad su realidad. Esto no significa que los sistemas se despreocupen de los problemas de la calidad educativa. Por el contrario, la exigencia planteada para una gestión de apoyo oportuno, flexible, ágil, eficaz y diversificado a las escuelas por parte del sistema es, desde mi punto de vista, quizás la recomendación de política educativa que más evidentemente emana de este campo de investigación. 
